\title{
Dosage de l'hémoglobine urinaire par un réactif au 3,3'-diméthylbenzidine : mise au point technique
}

\author{
Moutawakilou GOMINA ASSOUMANOU * et Simon A. AKPONA \\ UERS de Biochimie et de Biologie Moléculaire, Faculté de Médecine, Université de Parakou, BP : 123 \\ Parakou (République du Bénin). \\ *Auteur correspondant, E-mail : elboutraguero@yahoo.fr; Tél : + 22997107779
}

\section{RESUME}

La mesure de la teneur en hémoglobine dans les milieux biologiques relève de plusieurs techniques. L'objectif de ce travail était de déterminer la teneur en hémoglobine des urines lors d'une hémolyse intra vasculaire par un réactif au 3,3'-diméthylbenzidine. A partir d'une solution d'hémoglobine humaine dont la concentration a été déterminée par le réactif de Drabkin, une méthode en point final utilisant le 3,3'diméthylbenzidine comme chromogène en présence de $\mathrm{H}_{2} \mathrm{O}_{2}$ a été évaluée par sa sensibilité, sa répétabilité, sa reproductibilité et l'interférence de la bilirubine. Elle a ensuite été utilisée pour doser l'hémoglobine dans les urines de 56 malades présentant une hémolyse intra vasculaire aiguë. La linéarité de la méthode était bonne entre 0,06 et $1 \mathrm{~g} / \mathrm{L}$ d'hémoglobine; sa limite inférieure de détection était de $3 \mathrm{mg} / \mathrm{L}$ d'hémoglobine; la bilirubine n'interférait pas sur la méthode à la concentration de $100 \mathrm{mg} / \mathrm{L}$. La répétabilité (coefficient de variation intra essai $=3,12 \%, 2,36 \%$ et $1,27 \%$ respectivement pour des solutions d'hémoglobine de concentration $0,25,0,5$ et $1 \mathrm{~g} / \mathrm{L}$ ) et la reproductibilité (coefficient de variation inter essai $=4,15 \%, 3,12 \%$ et $1,67 \%$ respectivement pour des solutions d'hémoglobine de concentration $0,25,0,5$ et $1 \mathrm{~g} / \mathrm{L}$ ) pour 20 essais étaient bonnes. Cette méthode peut être utilisée en pratique courante quotidienne pour le dosage de l'hémoglobine urinaire dans les laboratoires de chimie clinique.

(C) 2011 International Formulae Group. All rights reserved.

Mots clés : hémoglobinurie, $o$-tolidine, répétabilité, reproductibilité.

\section{INTRODUCTION}

L'anémie hémolytique résultant d'une destruction prématurée des érythrocytes est un motif d'hospitalisation relativement fréquent surtout dans les services de pédiatrie (Dionne, 2006 ; Janus et Moerschel, 2010). De causes multiples, l'hémolyse intra vasculaire se manifeste par la triade anémieictère-splénomégalie (Arruda et al., 2010) et par un signe urinaire majeur, «les urines de couleur coca-cola», conséquence de l'hémoglobinurie. Si le syndrome hémolytique passe parfois inaperçu, dans certains cas il peut être sévère avec des séquelles cliniques redoutables (Rother et al., 2005). Le diagnostic positif de l'hémolyse intra vasculaire repose sur la mise en évidence de l'hémoglobine dans le plasma et les urines (Chalmers et Snell, 1993; Dionne, 2006). La mesure de la teneur en 
hémoglobine dans ces milieux biologiques relève de plusieurs techniques: spectrophotométrie directe (Wians et al., 1988), spectrophotométrie dérivée (Taulier et al., 1986; Copeland et al., 1989), chimiluminescence (Olsson et al., 1982), turbidimétrie (Engler et al., 1979) et colorimétrie (Ahlquist et Schwartz, 1975; Slaunwhite et al., 1979; Fairbanks et al., 1992). Les méthodes colorimétriques, plus usuelles, se font sur le plasma et mettent à profit l'activité peroxydasique de l'hémoglobine qui oxyde un chromogène (Fairbanks et al., 1992 ; Morgan et al., 1994; Dionne, 2006). La benzidine, premier chromogène utilisé s'est révélée cancérigène (Kum-Tatt et Ling, 1969 ; Morgan et al., 1994 ); elle a été alors remplacée par ses dérivés qui le sont moins comme l'attestent plusieurs études (Lijana et Williams, 1979; Levinson et Goldman, 1982 ; Pillier-Loriette et al., 1983 ; Takayanagi et Yashiro, 1984).

Les techniques de dosage de l'hémoglobine ont des limites en raison de substances interférentes qui peuvent être contenues dans les milieux biologiques (Fairbanks et al., 1992; Chalmers et Snell, 1993 ; Goyal et Basak, 2009).

$\mathrm{Si}$ le dosage de l'hémoglobine plasmatique se fait couramment dans les laboratoires de chimie clinique, la recherche de l'hémoglobine urinaire n'est le plus souvent réalisée que par la méthode qualitative utilisant des bandelettes réactives.

L'objectif de ce travail était de développer une technique colorimétrique simple, pratique et peu onéreuse de détermination quantitative de l'hémoglobine urinaire par un réactif au 3,3'diméthylbenzidine (o-tolidine) chez des sujets présentant une hémolyse intra vasculaire.

\section{MATERIEL ET METHODES}

\section{Cadre d'étude}

Cette étude a été réalisée dans le laboratoire de Biochimie du Centre Hospitalier Départemental du Borgou (CHDB) en République du Bénin.

\section{Matériel}

Les réactifs suivants ont été utilisés dans ce travail : acide acétique glacial $\left(\mathrm{CH}_{3} \mathrm{COOH}\right) 100 \%, \mathrm{MM}=60,05$, référence 310 K19356463, Merck; cyanure de potassium $(\mathrm{KCN})$ pur à $99 \%, \mathrm{MM}=65,12$, référence $\mathrm{P} / 4600 / 50$, Fisher Chemicals ; éthanol $\left(\mathrm{CH}_{3} \mathrm{CH}_{2} \mathrm{OH}\right)$ à $95^{\circ}$; ferricyanure de potassium $\left(\mathrm{C}_{6} \mathrm{FeK}_{3} \mathrm{~N}_{6}\right)$ pur à $99 \%, \mathrm{MM}=$ 329,26, référence $\mathrm{P} / 4880 / 50$, Fisher Chemicals ; hydrogénocarbonate de sodium $\left(\mathrm{HCO}_{3} \mathrm{Na}\right)$ pur pour analyse $99 \%, \mathrm{MM}=$ 84,01, référence S/4200/60, Fisher Chemicals ; 3,3'-diméthylbenzidine (otolidine) $\left(\mathrm{C}_{14} \mathrm{H}_{16} \mathrm{~N}_{2}\right), \mathrm{MM}=212,29$, Merck; peroxyde d'hydrogène $\left(\mathrm{H}_{2} \mathrm{O}_{2}\right)$ à $3 \%, \mathrm{MM}=$ 33,01, Lot: 000042, Cooper ; bandelettes réactives urinaires Taytec 10 parameters, référence : TY1115-0100, Lot : U10020205 (www.taytec.ca).

Nous avons utilisé un spectrophotomètre UV-Visible de marque SHIMADZU 1240 capable de lire les densités optiques à trois chiffres après la virgule.

\section{Méthodes}

\section{Préparation des solutions de travail}

Réactif $R 1$ : il a été préparé par mélange de un volume d'acide acétique glacial avec quatre volumes d'éthanol à $95^{\circ}$. Soit pour $500 \mathrm{ml}$ de R1 nous avons mélangé $100 \mathrm{ml}$ d'acide acétique glacial à $400 \mathrm{ml}$ de méthanol à $95^{\circ}$.

Réactif R2 (Solution mère de 3,3'diméthylbenzidine à $0,2 \mathrm{~g} / \mathrm{L}$ ): il a été obtenu en dissolvant 2 grammes de 3,3'- 
diméthylbenzidine dans $100 \mathrm{ml}$ de R1. Cette solution a été conservée dans un flacon brun à l'abri de la lumière et est restée stable pendant 45 jours à $4{ }^{\circ} \mathrm{C}$.

Réactif $R 3$ : il a été obtenu par une dilution au $1 / 5^{\text {ème }}$ du réactif $\mathrm{R} 2$ pour obtenir une solution de concentration $0,04 \mathrm{~g} / \mathrm{L}$. Cette solution est restée stable à la température du laboratoire $\left(20-25^{\circ} \mathrm{C}\right)$ pendant 24 heures.

Réactif $\mathrm{R} 4$ (Solution de peroxyde d'hydrogène, $\mathrm{H}_{2} \mathrm{O}_{2}$ à 2\% ) : il a été obtenu par dilution au 2/3 de la solution de $\mathrm{H}_{2} \mathrm{O}_{2}$ à $3 \%$ avec de l'eau distillée. Cette solution est restée stable entre 6 et 8 heures d'horloge.

Solution d'hémoglobine: elle a été préparée selon la méthode décrite par Fairbanks et al. (1992) à partir de $5 \mathrm{ml}$ de sang prélevé dans un tube contenant de l'EDTA par ponction veineuse superficielle. L'échantillon de sang obtenu a été centrifugé à $4000 \mathrm{rpm}$ pendant 10 minutes et le plasma décanté. Le culot globulaire $(2 \mathrm{ml})$ a été resuspendu dans $3 \mathrm{ml}$ de sérum salé $0,9 \%$ et centrifugé à $4000 \mathrm{rpm}$ pendant 15 minutes. Cette opération a été répétée trois fois. Les globules rouges ainsi lavés ont été lysés avec de l'eau distillée pendant 1 heure $(100 \mu 1$ de globules rouges dans $3 \mathrm{ml}$ d'eau distillée) puis le mélange centrifugé à $4500 \mathrm{rpm}$ pendant 30 minutes. Le surnageant de centrifugation constituait la solution d'hémoglobine.

Réactif de Drabkin : il a été obtenu en pesant dans une fiole jaugée de $1000 \mathrm{ml}, 1$ gramme d'hydrogénocarbonate de sodium, $52 \mathrm{mg}$ de cyanure de potassium, $198 \mathrm{mg}$ de ferricyanure de potassium; nous y avons complété de l'eau distillée pour obtenir une solution de $1000 \mathrm{ml}$. Cette solution est stable pendant 15 jours à la température du laboratoire $\left(20-25^{\circ} \mathrm{C}\right)$.

\section{Détermination de la concentration de la solution d'hémoglobine}

La mesure de la concentration de la solution d'hémoglobine a été effectuée par la méthode de Drabkin (1946). A $5 \mathrm{ml} \mathrm{du}$ réactif de Drabkin, nous avons ajouté $20 \mu \mathrm{l}$ de la solution d'hémoglobine dans un tube à essai. Après homogénéisation du mélange, nous avons laissé incuber 20 minutes à la température du laboratoire et avons lu la densité optique au spectrophotomètre à 540 $\mathrm{nm}$ contre un blanc réactif constitué de la solution de Drabkin. La densité optique lue a été reportée sur une courbe d'étalonnage réalisée préalablement pour déterminer la concentration en hémoglobine de la solution.

\section{Etude de la cinétique du 3,3'- diméthylbenzidine en présence de l'hémoglobine}

L'étude de la cinétique du 3,3'diméthylbenzidine a été réalisée en présence de concentrations différentes en hémoglobine. A partir d'une solution mère d'hémoglobine de concentration $1 \mathrm{~g} / \mathrm{L}$ (16 $\mu \mathrm{mol} / \mathrm{L})$ déterminée par la méthode de Drabkin, nous avons réalisé des dilutions successives de raison $1 / 2$. Les concentrations des solutions d'hémoglobine utilisées étaient : $1,0,5$ et $0,25 \mathrm{~g} / \mathrm{L}$.

Dans des tubes à hémolyse, nous avons introduit $1000 \mu \mathrm{l}$ de la solution R3, $1000 \mu \mathrm{l}$ de la solution R4 et laissé au repos pendant 5 minutes; puis nous avons ajouté par tube $10 \mu \mathrm{l}$ de chaque dilution de la solution d'hémoglobine et vortexé. L'évolution de la densité optique dans chaque tube a été suivie dès le déclenchement de la réaction par la solution d'hémoglobine au spectrophotomètre à $630 \mathrm{~nm}$ toutes les 30 secondes pendant 5 minutes dans une cuve de 1 centimètre de trajet optique contre un blanc réactif constitué du mélange des solutions $\mathrm{R} 3$ et R4 (1: $1 ; \mathrm{V} / \mathrm{V})$. 


\section{Réalisation de la courbe d'étalonnage}

La courbe d'étalonnage a été réalisée à partir d'une solution d'hémoglobine de concentration $1 \mathrm{~g} / \mathrm{L}(16 \mu \mathrm{mol} / \mathrm{L})$ déterminée par la méthode de Drabkin et diluée à la raison $1 / 2$. Dans des tubes à hémolyse, nous avons introduit $1000 \mu 1$ de la solution R3, $1000 \mu$ l de la solution R4 et nous avons laissé au repos pendant 5 minutes (le mélange réactionnel avait un $\mathrm{pH}$ de 3-4); puis nous avons ajouté par tube $10 \mu \mathrm{l}$ de chaque dilution de la solution d'hémoglobine. Après incubation de 10 minutes à la température du laboratoire $\left(20-25{ }^{\circ} \mathrm{C}\right)$, la densité optique de la coloration obtenue a été lue dans une cuve de 1 centimètre de trajet optique au spectrophotomètre à $630 \mathrm{~nm}$ contre un blanc réactif constitué du mélange des solutions $\mathrm{R} 3$ et R4 $(1: 1 ; \mathrm{V} / \mathrm{V})$; ce qui nous a permis de tracer la courbe d'étalonnage (densités optiques ou absorbances en fonction des concentrations des solutions d'hémoglobine).

Evaluation de la méthode de dosage de l'hémoglobine urinaire par le réactif au 3,3'-diméthylbenzidine

Elle a été effectuée en utilisant les éléments du protocole de validation de techniques (Valtec) 97 de la Société Française de Biologie Clinique (Capolaghi et al., 1997).

La sensibilité : elle a été évaluée par la mesure des concentrations différentes de solutions d'hémoglobine obtenues à partir de dilutions d'une solution d'hémoglobine de 3 $\mathrm{g} / \mathrm{L}$ (48 $\mu \mathrm{mol} / \mathrm{L})$. Ce qui a permis de déterminer la limite inférieure de détection de la méthode.

La répétabilité : elle a été évaluée par le coefficient de variation intra essai avec des solutions d'hémoglobine de concentration $0,25,0,5$ et $1 \mathrm{~g} / \mathrm{L}$. Vingt (20) dosages répétés on été réalisés avec chacune des trois solutions d'hémoglobine selon la modalité décrite plus haut. La moyenne des concentrations obtenues a été calculée de même que son écart-type et le coefficient de variation intra essai.

La reproductibilité: elle a été appréciée par le coefficient de variation inter essai avec des solutions d'hémoglobine de concentration $0,25,0,5$ et $1 \mathrm{~g} / \mathrm{L}$ en reprenant les dosages effectués pour évaluer la répétabilité, une semaine après en changeant de conditions (autre technicien, température du laboratoire : $20^{\circ} \mathrm{C}$ et $30{ }^{\circ} \mathrm{C}$ ).

\section{Etude de l'effet de la bilirubine sur l'absorbance à $630 \mathrm{~nm}$}

L'interférence de la bilirubine sur l'absorbance de la coloration obtenue à 630 $\mathrm{nm}$ a été étudiée en utilisant un sérum ictérique de concentration en bilirubine totale de $200 \mathrm{mg} / \mathrm{L}$. Dans des tubes à hémolyse, nous avons introduit $500 \mu 1$ de la solution R3, $500 \mu 1$ de la solution R4 et nous avons laissé au repos pendant 5 minutes; puis nous avons ajouté par tube $1000 \mu \mathrm{l}$ de solution de bilirubine à $200 \mathrm{mg} / \mathrm{L}$ ou diluée pour obtenir des concentrations totales en bilirubine du mélange de 100, 90, 80, 70, 60, 50, 40, 30, $20,10 \mathrm{mg} / \mathrm{L}$ et $1000 \mu \mathrm{l}$ d'eau distillée dans un tube témoin. La réaction a été déclenchée par ajout de $10 \mu \mathrm{l}$ de solution d'hémoglobine à $0,5 \mathrm{~g} / \mathrm{L}$ dans chaque tube ainsi préparé. Après 10 minutes d'incubation, la densité optique de la coloration obtenue à été lue au spectrophotomètre à $630 \mathrm{~nm}$, dans une cuve de 1 centimètre de trajet optique. Le blanc réactif était constitué du mélange des solutions R3 et R4 (1: 1 ; V/V).

\section{Dosage de l'hémoglobine urinaire par le réactif au 3,3'-diméthylbenzidine \\ Il a été réalisé à partir d'urines fraichement émises par des malades présentant une hémolyse intra vasculaire aiguë provenant d'une part des services de}


Pédiatrie et de Médecine Interne du CHD-B et, d'autre part, des formations sanitaires de la ville de Parakou. Vingt (20) millilitres de ces urines ont été recueillies dans des flacons stériles et acheminées dans l'heure qui suit au laboratoire de biochimie du CHD-B. Le dosage de l'hémoglobine dans chaque échantillon d'urine a été réalisé selon le protocole de mesure retenu. Cette opération a été réalisée deux fois sur le même échantillon d'urine et la moyenne des densités optiques obtenues a été utilisée. Les densités optiques obtenues ont été reportées sur la courbe d'étalonnage pour déterminer les concentrations correspondantes en hémoglobine de l'échantillon d'urine.

Détermination qualitative de certains paramètres des échantillons d'urines par la bandelette réactive Taytec 10 parameters

Les paramètres suivants ont été déterminés par la bandelette réactive Taytec 10 parameters sur chaque échantillon d'urine : nitrites (pour éliminer une infection des voies urinaires pouvant donner des faux positifs), densité (pour contrôler la limite de linéarité de la méthode), $\mathrm{pH}$ (pour contrôler son influence sur la cinétique), bilirubinurie et hémoglobinurie. Selon les recommandations du fabricant, nous avons trempé dans chaque échantillon d'urine une bandelette réactive et nous avons comparé les colorations apparues à une échelle de couleur après une durée qui était de 60 secondes pour les nitrites, le $\mathrm{pH}$ et l'hémoglobine, de 45 secondes pour la densité et de 30 secondes pour la bilirubine.

\section{Analyse statistique}

Les données obtenues ont été analysées à l'aide des logiciels SPSS Statistics 17.0 (IBM) et Excel 2007. Les graphiques ont été obtenus en utilisant les mêmes logiciels. Le calcul des moyennes et des écarts-types nous a permis d'évaluer la précision de la méthode.

\section{RESULTATS}

La Figure 1 montre la cinétique de la transformation du 3,3'-diméthylbenzidine par trois concentrations différentes d'hémoglobine en fonction du temps à la température du laboratoire $\left(20-25{ }^{\circ} \mathrm{C}\right)$. La Figure 2 présente les résultats de l'étude de la linéarité de la méthode en point final avec le réactif au 3,3'-diméthylbenzidine (densités optiques à $630 \mathrm{~nm}$ en fonction de différentes concentrations de solutions d'hémoglobine). La Figure 3 montre l'influence de la bilirubine sur la mesure de la densité optique à $630 \mathrm{~nm}$ de la coloration issue de l'oxydation du 3,3'-diméthylbenzidine par le peroxyde d'hydrogène en présence d'hémoglobine. Le Tableau 1 présente les éléments de la précision de la méthode en point final du dosage de l'hémoglobine urinaire par le réactif au 3,3'diméthylbenzidine. La durée de la stabilité de la coloration obtenue était de quarante deux (42) minutes. La limite inférieure de détection de la méthode était de $3 \mathrm{mg} / \mathrm{L}$ $(0,048 \mu \mathrm{mol} / \mathrm{L})$. Les valeurs extrêmes de l'hémoglobine déterminées dans les échantillons d'urine par le réactif au 3,3'diméthylbenzidine étaient de 0,068 et 0,89 $\mathrm{g} / \mathrm{L}$. La bilirubinurie était négative de même que la détermination des nitrites par la méthode aux bandelettes réactives urinaires. Le pH moyen des urines était de 6,46 $\pm 1,16$ et la densité urinaire moyenne de 1,015 \pm 0,005 . L'hémoglobinurie était positive à ++ pour $42 \%$ des échantillons d'urine et à +++ pour $58 \%$ des échantillons à la bandelette réactive urinaire. 


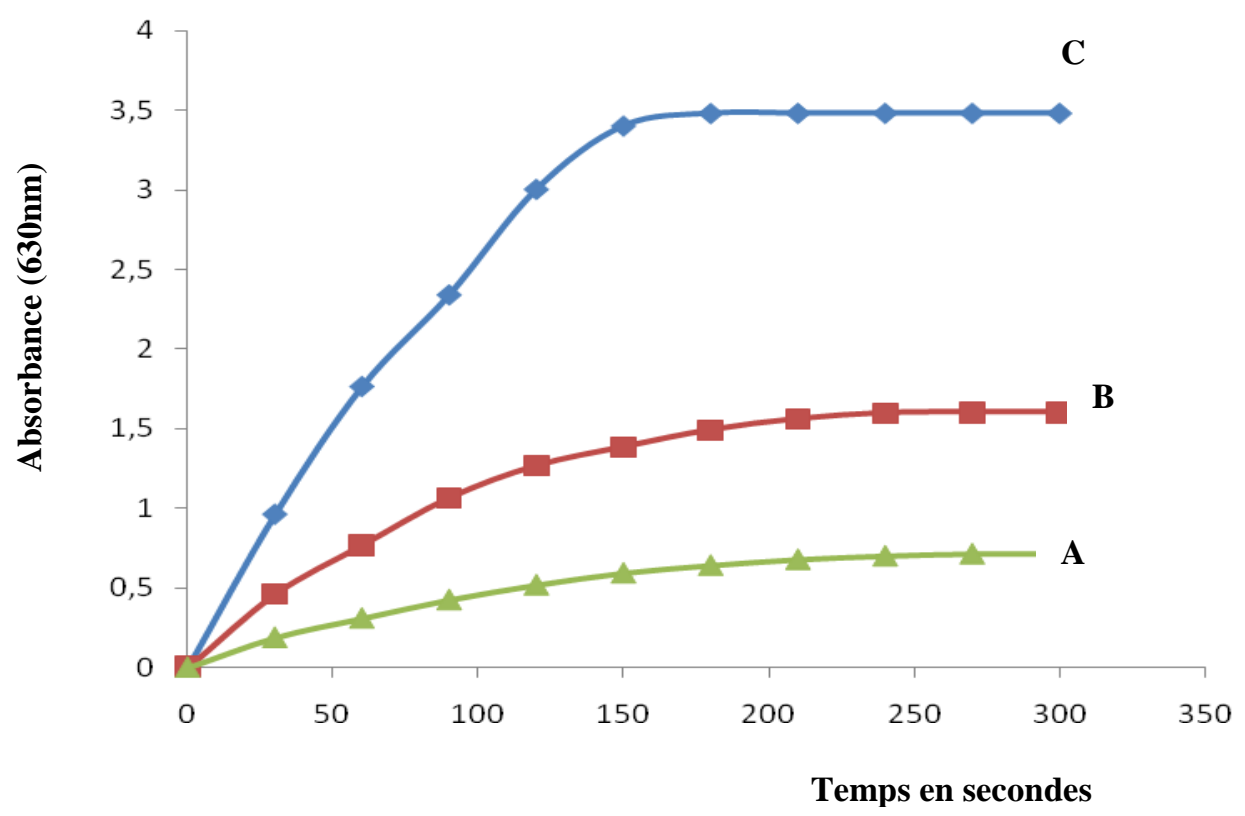

Figure 1 : Evolution de la densité optique (absorbance) en fonction du temps en présence de trois concentrations différentes d'hémoglobine.

A : Concentration en hémoglobine $=0,25 \mathrm{~g} / \mathrm{L} ; \mathrm{B}:$ Concentration en hémoglobine $=0,5 \mathrm{~g} / \mathrm{L} ; \mathrm{C}:$ Concentration en hémoglobine $=1 \mathrm{~g} / \mathrm{L}$

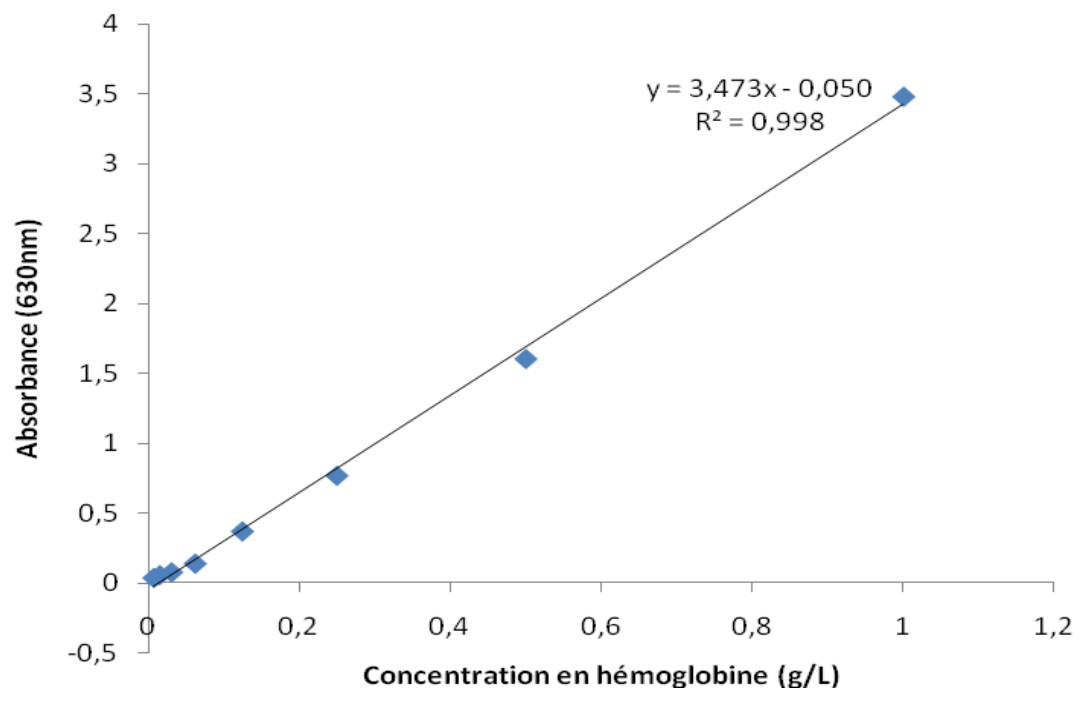

Figure 2 : Etude de la linéarité de la méthode : courbe d'étalonnage. 


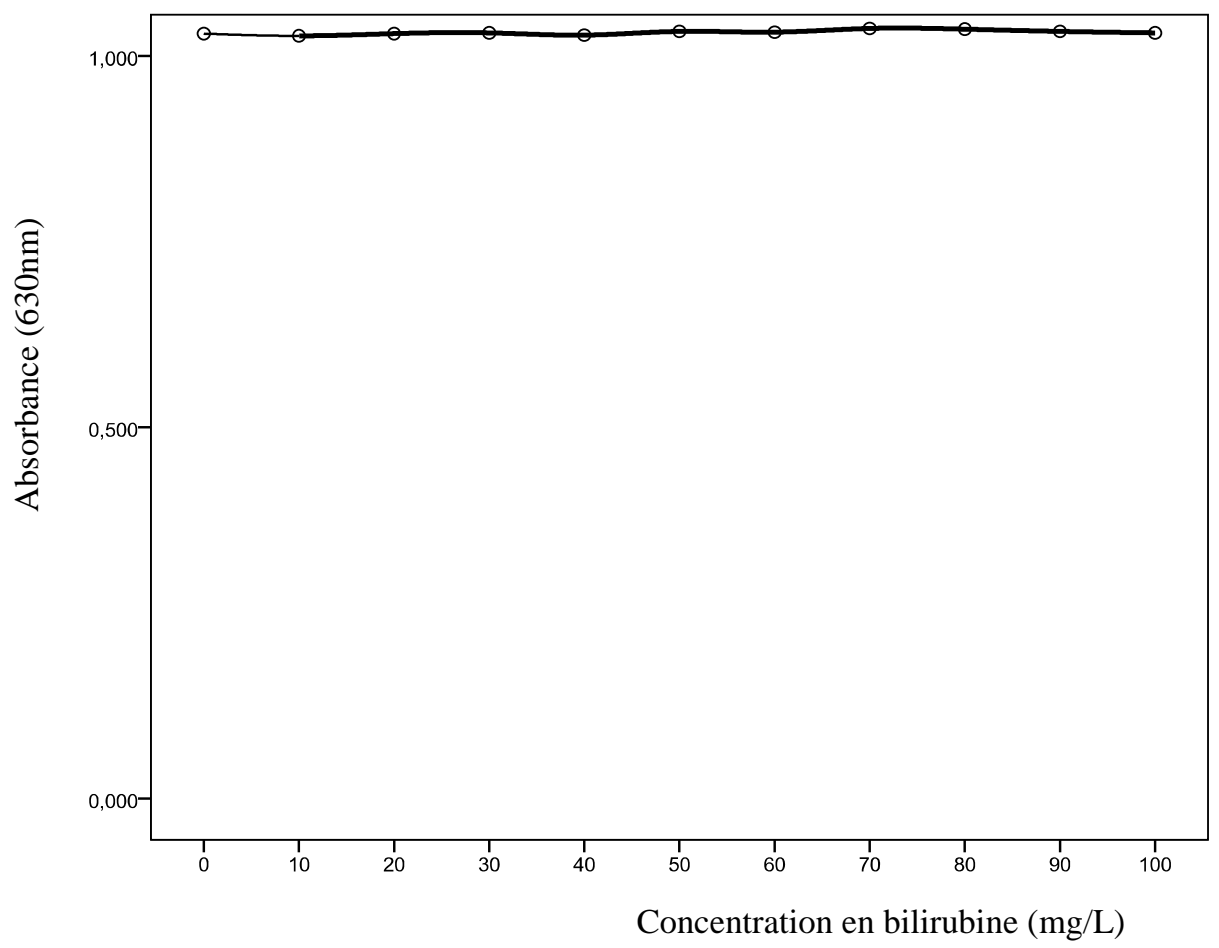

Figure 3 : Interférence de la bilirubine sur la mesure de la densité optique.

Tableau 1 : Précision de la méthode de dosage de l'hémoglobine urinaire.

\begin{tabular}{lccc}
\hline & \multicolumn{3}{c}{ Concentration en hémoglobine $(\mathbf{g} / \mathbf{L})$} \\
\cline { 2 - 4 } & $\mathbf{0 , 2 5}$ & $\mathbf{0 , 5}$ & $\mathbf{1}$ \\
\hline CVintra, \% $(\mathbf{n}=\mathbf{2 0})$ & 3,12 & 2,36 & 1,27 \\
CVinter, \% $(\mathbf{n = 2 0})$ & 4,15 & 3,12 & 1,67 \\
\hline
\end{tabular}

CVintra : coefficient de variation intra essai ; CVinter : coefficient de variation inter essai

Tableau 2 : Coefficients de variation inter essai de la méthode au 3,3'-diméthylbenzidine et de quatre méthodes de dosage de l'hémoglobine plasmatique.

\begin{tabular}{lcc}
\hline & \multicolumn{2}{c}{ Coefficients de variation inter essai \% } \\
\cline { 2 - 3 } & Hémoglobine $=\mathbf{0 , 5} \mathbf{~ g / L}$ & Hémoglobine $\mathbf{= 1} \mathbf{~ g / L}$ \\
\hline 3,3'-diméthylbenzidine $(\mathbf{n}=\mathbf{2 0})$ & 3,1 & 1,7 \\
Harboe $(\mathbf{1 9 5 9})(\mathbf{n}=\mathbf{5 6})$ & 0,8 & 0,3 \\
Noe et al. (1984) $(\mathbf{n}=\mathbf{5 6})$ & 0,9 & 0,4 \\
Blakney et Dinwoodie $(\mathbf{1 9 7 5})(\mathbf{n = 3 5})$ & 1,8 & 1,0 \\
Kahn et al. (1981) $(\mathbf{n}=\mathbf{5 6})$ & 0,5 & 1,0 \\
\hline
\end{tabular}




\section{DISCUSSION}

Nous avons utilisé une méthode quantitative en point final, comme Levinson et Goldman (1982), Pillier-Loriette (1983), basée sur les propriétés chromogéniques du 3,3'-diméthylbenzidine pour doser l'hémoglobine dans l'urine au cours d'un syndrome hémolytique après évaluation de ladite méthode.

Limites de la méthode : la spécificité de la méthode peut être compromise car cette technique peut mettre en évidence des substances ayant une activité peroxidaselike telles que la myoglobine, le cytochrome, la catalase, la peroxydase (Levere et al., 1971) ou la présence de peroxydases produites par les bactéries lors d'une infection des voies urinaires (Yoder et al., 1965; Free et Free, 1972). Pour éviter ces causes d'erreur, nous avons travaillé sur des urines fraichement émises, prélevées dans des flacons stériles et pour lesquelles le test aux nitrites était négatif.

La limite inférieure de détection de la méthode au 3,3'-diméthylbenzidine utilisée dans ce travail était de $3 \mathrm{mg} / \mathrm{L}(0,048$ $\mu \mathrm{mol} / \mathrm{L}$ ), résultat identique à celui trouvé par Chalmers et Snell (1993) qui est également de $3 \mathrm{mg} / \mathrm{L}$ d'hémoglobine dans le plasma et l'urine en utilisant comme chromogène la chlorpromazine. Par contre, Takayanagi et Yashiro (1984) trouvent une limite inférieure de détection de $20 \mathrm{mg} / \mathrm{L}$ si le chromogène est l'ABTS.

L'étude de la cinétique de la transformation du 3,3'-diméthylbenzidine par des concentrations différentes d'hémoglobine en fonction du temps à la température du laboratoire $\left(20-25^{\circ} \mathrm{C}\right)$ a montré une cinétique croissante rapide pendant 150 secondes, suivi de son ralentissement progressif pour atteindre la valeur maximale au bout de 250 secondes pour des concentrations en hémoglobine de 0,25 et $0,5 \mathrm{~g} / \mathrm{L}$ courbes $\mathrm{A}$ et $\mathrm{B}$ respectivement (Figure 1). A $1 \mathrm{~g} / \mathrm{L}$ d'hémoglobine, la vitesse maximale de la réaction a été atteinte au bout de 150 secondes (courbe C, Figure 1). Ce résultat nous a permis de fixer la durée d'incubation à 10 minutes à la température du laboratoire $\left(20-25{ }^{\circ} \mathrm{C}\right)$ pour notre méthode. Il s'agissait d'une méthode en point final utilisée dans ce travail pour assurer une bonne praticabilité. Les méthodes en point final décrites dans la littérature utilisent une durée d'incubation qui varie entre 3 et 90 minutes (Levinson et Goldman, 1982; Takayanagi et Yashiro, 1984 ; Chalmers et Snell, 1993). La durée d'incubation dans notre méthode, 10 minutes, est donc courte. Cela offre un avantage certain dans la durée de sa réalisation en pratique quotidienne courante.

La linéarité de la méthode a été étudiée avec des solutions d'hémoglobine de concentrations différentes. Cette linéarité était bonne entre 0,06 et $1 \mathrm{~g} / \mathrm{L}$ d'hémoglobine mais mauvaise pour des concentrations en hémoglobine inférieures (Figure 2). En utilisant la méthode en point final et le tétraméthylbenzidine comme chromogène, Levinson et Goldman (1982) trouvent une bonne linéarité pour des concentrations en hémoglobine de 0,02 à $2,5 \mathrm{~g} / \mathrm{L}$. Pour Goyal et Basak (2009) la bonne linéarité se situe entre 6 et $400 \mathrm{mg} / \mathrm{L}$ d'hémoglobine en utilisant la méthode cinétique avec l'o-tolidine comme chromogène.

Nous n'avons pas noté d'interférence de la bilirubine sur la mesure de la densité optique de la coloration issue de l'oxydation du 3,3'-diméthylbenzidine par l'hémoglobine en présence du peroxyde d'hydrogène, même à la concentration en bilirubine de $100 \mathrm{mg} / \mathrm{L}$ (Figure 3). La même observation est rapportée par Levinson et Goldman (1982), Mezzour et al. (2006) avec le tétraméthylbenzidine, Chalmers et Snell (1993) avec la chlorpromazine comme chromogène en utilisant la bilirubine à des concentrations plus élevées que les nôtres $(250 \mathrm{mg} / \mathrm{L})$.

La répétabilité : elle a été déterminée sur 20 dosages répétés de trois concentrations différentes d'hémoglobine et exprimée par le coefficient de variation intra essai. Les 
coefficients de variation intra essai de $3,12 \%$, $2,36 \%$ et $1,27 \%$ obtenus pour des concentrations en hémoglobine respectivement de $0,25,0,5$ et $1 \mathrm{~g} / \mathrm{L}$ montrent une précision plus importante pour des concentrations en hémoglobine élevées (Tableau 1). Ces valeurs, inférieures à $5 \%$ sont le témoin d'une bonne précision comme l'ont indiqué Mezzour et al. (2006) qui trouvent une répétabilité de $2,43 \%$ et $2,03 \%$ sur 60 dosages pour des concentrations en hémoglobine respectives de $0,14 \mathrm{~g} / \mathrm{L}$ et 0,4 $\mathrm{g} / \mathrm{L}$.

La reproductibilité : elle a été déterminée sur une série de 20 dosages répétés à une semaine d'intervalle, dans des conditions différentes, de trois concentrations différentes d'hémoglobine et exprimée par le coefficient de variation inter essai. Les valeurs obtenues $(4,15 \%, 3,12 \%, 1,67 \%)$ étaient conformes aux recommandations de Vassault et al. (1999) qui rapportent que le coefficient de variation de la reproductibilité doit être de 1,33 fois le coefficient de variation de la répétabilité. Mezzour et al. (2006) trouvent des coefficients de variation inter essai de $2,95 \%$ et $3,17 \%$ respectivement pour des solutions d'hémoglobine de concentration $0,14 \mathrm{~g} / \mathrm{L} \quad$ et $0,4 \mathrm{~g} / \mathrm{L}$ avec le tétraméthylbenzidine comme chromogène et pour 60 dosages. Le Tableau 2 montre les valeurs du coefficient de variation inter essai de deux concentrations d'hémoglobine de la méthode utilisée dans ce travail et de quatre méthodes décrites dans la littérature. De ce tableau, il ressort que le coefficient de variation inter essai est meilleur lorsque la concentration en hémoglobine augmente sauf pour la méthode de Kahn et al. (1981). Cette observation pourrait s'expliquer par le fait que plus la concentration de la solution d'hémoglobine mesurée par une méthode est élevée mieux elle se trouve dans la zone de linéarité de la courbe d'étalonnage de la méthode.

La détermination de quelques paramètres urinaires : la recherche de nitrites par la méthode aux bandelettes réactives urinaires a permis d'éliminer une infection urinaire qui pourrait constituer une cause de faux positif à la méthode au 3,3'diméthylbenzidine ou la majorer. Tous les échantillons testés étaient négatifs aux nitrites témoins d'urines non infectées par des bacilles à Gram négatif. De même, la densité moyenne et le $\mathrm{pH}$ moyen des échantillons d'urine analysés qui étaient respectivement de 1,015 \pm 0,005 et $6,06 \pm 1,16$ montrent que ces urines avaient des caractéristiques physicochimiques normales.

La technique mise au point : elle a été de réalisation facile et n'a nécessité aucun équipement spécialisé. Les réactifs indispensables étaient disponibles à moindre coût et la durée de réalisation de la technique, 10 minutes environ, est compatible avec une bonne praticabilité. La stabilité de la coloration, 42 minutes, a permis de travailler sur une série de 14 échantillons d'urine. La quantité d'urine, $10 \mu 1$, dans un volume réactionnel de $2010 \mu \mathrm{l}$, ne modifiera pas la mesure de la densité optique quelles que soient la densité et le $\mathrm{pH}$ des urines testées.

Nous avons réalisé dans ce travail la mise au point d'une méthode quantitative en point final de dosage de l'hémoglobine urinaire par un chromogène, le 3,3'diméthylbenzidine. L'évaluation de ladite méthode par sa sensibilité, sa répétabilité et sa reproductibilité a montré des résultats qui permettent de l'adopter en pratique de chimie clinique courante. De ce qui précède nous suggérons que les laboratoires de chimie clinique s'approprient cette méthode pour le dosage quantitatif de l'hémoglobine dans l'urine au cours des hémolyses intra vasculaires. 


\section{REMERCIEMENTS}

Nous remercions le Conseil

Scientifique de l'Université de Parakou qui a financé ce travail.

\section{REFERENCES}

Ahlquist DA, Schwartz S. 1975. Use of leucodyes in the quantitative colorimetric microdetermination of hemoglobin and other heme compounds. Clin. Chem., 21: 362-369.

Arruda MM, Rodrigues CA, Yamamoto M, Figueiredo MS. 2010. Paroxysmal nocturnal hemoglobinuria: from physiopathology to treatment. Rev. Assoc. Med. Bra., 56(2): 214-221.

Blakney GB, Dinwoodies AJ. 1975. Spectrophotometric scanning technique for the rapid determination of plasma hemoglobin. Clin. Biochem., 8: 96-102.

Capolaghi B, Vassault A, Grafmeyer D, Yvert JP. 1997. Le protocole Valtec: évolution du concept et du contenu. Ann. Biol. Clin., 55(2): 167-173.

Chalmers AH, Snell LE. 1993. Estimation of plasma and urinary hemoglobin by a rate spectrophotometric method. Clin. Chem., 39(8): 1679-1682.

Copeland BE, Dyer PJ, Pesce AJ. 1989. Hemoglobin determination in plasma or serum by first derivative recording spectrophotometry: evaluation of the procedure of Soloni, Cunningham, and Amazon. Am. J. Clin. Pathol., 92: 619624.

Dionne JL. 2006. L'anémie hémolytique: approche générale. Ann. Biol. Clin., 43(2): 34-40.

Drabkin DL. 1946. Spectrophotometric studies -XIV. The crystallographic and optical properties of the haemoglobin of man, in comparison with those of other species. J. Biol. Chem., 164: 703.

Engler R, Pointis J, Rondeau Y. 1979. Détermination immunochimique de l'hémoglobine humaine dans les liquides biologiques. Clin. Chem., 25: 497-499.

Fairbanks VF, Ziesmer SC, O'Brien PC. 1992. Methods for measuring plasma hemoglobin in micro molar concentration compared. Clin. Chem., 38(1): 132-140.

Free AH, Free HM. 1972. Urinalysis, critical discipline of clinical science. CRC Crit. Rev. Clin. Lab. Sci., 3(4): 481-531.

Goyal M M, Basak A. 2009. Estimation of plasma hemoglobin by modified kinetic method using 0-Tolidine. Indian Journal of Clinical Biochemistry, 24(1): 36-41.

Harboe MA. 1959. Method for determination of hemoglobin in plasma by nearultraviolet spectrophotometry. Scand. J. Clin. Lab. Invest., 11: 66-70.

Janus J, Moerschel SK. 2010. Evaluation of anemia in children. Am. Fam. Physician, 81(12): 1462-1471.

Kahn SE, Watkins BF, Bermes EWJr. 1981. An evaluation of a spectrophotometric scanning technique for measurement of plasma hemoglobin. Ann. Clin. Lab. Sci., 11: 126-131.

Kum-Tatt L, Ling AM. 1969. Determination of micro-quantities of haemoglobin in serum. Mikrochim Acta, 5: 995-1002.

Levere RD, Swerdlow F, Garavoy MR. 1971. Measurement of human plasma hemoglobin by difference spectrophotometry. J. Lab. Clin. Med., 77(1): 168-176.

Levinson SS , Goldman J. 1982. Measuring hemoglobin in plasma by reaction with tetramethylbenzidine. Clin. Chem., 28(3): 471-474.

Lijana RC, Williams MC. 1979. Tetramethylbenzidine- a substitute for benzidine in hemoglobin analysis. J. Clin. Lab. Med., 94(2): 266-276.

Mezzour H, Zerouale K, Neffati F, Douki W, Ben Amor A, Najjar MF. 2006. Évaluation d'une technique spectrophotométrique pour le dosage de 
l'hémoglobine plasmatique sur Konelab 30TM. Ann. Biol. Clin., 64(4): 319-326.

Morgan DL, Dunnick JK, Goehl T, Jokinen MP, Matthews HB, Zeiger E, Mennear JH. 1994. Summary of the national toxicology program benzidine dye initiative. Environmental Health Perspectives, 102(2): 63-78.

Noe DA, Weedn V, Bell WR. 1984. Direct spectrophotometry of serum hemoglobin: Allen correction compared with a threewavelength polychromatic analysis. Clin. Chem., 30: 627-630.

OlssonT, Bergström K, Thore A. 1982. A sensitive methodfor determination of serum hemoglobin based on iso-luminol chemiluminescence. Clin. Chim. Acta, 122: 125-133.

Pillier-Loriette C, Wingerter P, Gerota J, Schenmtzler C, Reviron J, Aymard P. 1983. Determination of plasma hemoglobin in fresh plasma for therapeutic use by the TBM $\left(3,3^{\prime}, 5,5^{\prime}\right.$ tetremethylbenzidine) method. Rev. Fr. Transfus. Immunohematol., 26(3): 299312.

Rother RP, Bell L, Hillmen P. 2005. The clinical sequelae of intravascular hemolysis and extracellular plasma hemoglobin: a novel mechanism of human disease. JAMA, 293(13): 16531662.

Slaunwhite D, Clement J, Tussay RL, Reynoso G. 1979. Leucomalachite green assay for free hemoglobin in serum. Am. J. Clin. Pathol., 72: 852-855.

Takayanagi $\mathrm{M}$ and Yashiro T. 1984. Colorimetry of hemoglobin in plasma with 2,2'-azino-di(3-ethylbenzthiazolone6-sulfonic acid) (ABTS). Clin. Chem., 30(3): 357-359.

Taulier A, Levilain P, Lemonnier A. 1986. Intérêt de la spectrophotométrie dérivée pour le dosage de l'hémoglobine plasmatique et urinaire: comparaison avec la méthode utilisant la correction d'Allen. Ann. Biol. Clin., 44: 242-248.

Vassault A, Grafmeyer D, De Graeve R, Cohen R, Beaudonnet A, Bienvenu J.1999. Quality specifications and allowable standards for validation of methods used in clinical biochemistry. Ann. Biol. Clin., 57(6): 685-695.

Wians FHJr, Miller CL, Heald JI, Clark H. 1988. Evaluation of a direct spectrophotometric procedure for quantitating plasma hemoglobin. Lab. Med., 19:151-155.

Yoder J, Adam EC, Free AH. 1965. Simultaneous screening for urinary occult blood, protein, glucose, and pH. Amer. J. Med. Tech., 31: 285. 